\title{
Efeito de fungicidas no cafeeiro conilon
}

\section{Thaisa Thomazini HERZOG ${ }^{1 *}$, Bruno Sérgio Oliveira e SILVA ${ }^{1}$, Marcelo Barreto da SILVA ${ }^{1}$,} Fábio Luiz PARTELLI ${ }^{1}$, Antônio Fernando de SOUZA ${ }^{2}$

\author{
${ }^{1}$ Programa de Pós-Graduação em Agricultura Tropical, Centro Universitário Norte do Espírito Santo, \\ Universidade Federal do Espírito Santo, São Mateus, ES, Brasil. \\ ${ }^{2}$ Instituto Federal de Educação, Ciência e Tecnologia do Espírito Santo, Santa Teresa, ES, Brasil. \\ *E-mail: thaisaherzog@agronoma.eng.br
}

Recebido em setembro/2017; Aceito em maio/2018.

\begin{abstract}
RESUMO: O objetivo foi avaliar o efeito de fungicidas no controle da ferrugem, no crescimento vegetativo e reprodutivo do café conilon. O delineamento experimental utilizado foi em blocos casualizados com seis tratamentos e quatro repetições (duas aplicações de piraclostrobina e epoxiconazol + boscalida e duas aplicações de piraclostrobina e epoxiconazol - T1; três aplicações de piraclostrobina e epoxiconazol - T2; três de piraclostrobina, epoxiconazol e fluxapiroxade - T3; três de epoxiconazol - T4 e três de piraclostrobina - T5 e, sem aplicação de fungicida - T6). Foram realizadas avaliações do progresso da ferrugem do cafeeiro; do crescimento de ramos, número de nós por ramo e comprimento dos internódios; índice relativo de clorofila; número médio de rosetas com frutos, de frutos por ramo e de frutos por roseta; uniformidade de maturação dos frutos; porcentagem de frutos chochos, peso dos frutos e produtividade. Com base nos resultados obtidos no trabalho, concluiu-se que a aplicação com epoxiconazol proporcionou menor crescimento acumulado e número de nós por ramo plagiotrópico e menor número de rosetas com frutos. Os tratamentos com piraclostrobina influenciaram positivamente os índices relativo de clorofila e proporcionaram valores inferiores da relação clorofila $a / b$. Não houve efeito dos produtos na cultura do café conilon nas demais variáveis monitoradas.
\end{abstract}

Palavras-chave: Coffea canephora, estrobilurinas, Hemileia vastatrix, respostas fisiológicas, triazóis.

\section{Effect of fungicides on conilon coffee}

\begin{abstract}
The aim of it was evaluate the effect of fungicides in control of rust, vegetative growth and reproductive conilon coffee. The experiment was design in radom blocks with six treatments and four replicates (two applications of pyraclostrobin and epoxiconazole + boscalid and two applications of pyraclostrobin and epoxiconazole - T1; three applications of pyraclostrobin and epoxiconazole - T2; three of pyraclostrobin, epoxiconazole and fluxapyroxad - T3; three of epoxiconazole - T4 and three of pyraclostrobin - T5 and, without application of fungicide - T6) and four replicates. Evaluations of rust progress were carried out; growth of branches, number of nodes per branch and length of internodes; relative index of chlorophyll; average number of rosettes with fruits, fruits per branch and fruits per rosette and productivity. Based on the results obtained in the work, it was concluded that the application with epoxiconazole provided lower cumulative growth and number of nodes per plagiotropic branch, and lower number of rosettes with fruits. The treatments with pyraclostrobin positively influenced the relative chlorophyll indices and provided lower values of the chlorophyll a/b ratio. There was no effect of the products in conilon coffee culture in the other monitored variables.
\end{abstract}

Keywords: Coffea canephora, strobilurins, Hemileia vastatrix, physiological responses, triazoles.

\section{INTRODUÇÃO}

O Espírito Santo destaca-se como o maior produtor de café conilon (Coffea canephora Pierre ex A. Froehner), respondendo por cerca de $63,0 \%$ da produção nacional (CONAB, 2017). Contudo, o cultivo do café conilon confere desafios aos produtores rurais, dentre eles a ferrugem (Hemileia vastatrix Berkeley \& Broome) principal doença da cultura em nível nacional (ZAMBOLIM et al., 2009), por ocasionar danos de até $47,0 \%$ na produção (CAPUCHO et al., 2013b).

Dentre as estratégias de controle efetivo das doenças, destaca-se a aplicação de fungicidas. Porém, com o lançamento de alguns princípios ativos, como do grupo das estrobilurinas, o controle químico ganha novas perspectivas, por também atuarem de forma positiva na fisiologia das plantas (VENÂNCIO et al., 2003). Baseados nos efeitos fisiológicos, proporcionados com o uso das estrobilurinas, outros fungicidas, de grupos químicos distintos, também estão sendo estudados, como as anilidas, as carboxamidas e os triazóis.

Em estudos feitos em diversas culturas, foram observados aumento da atividade da enzima nitrato redutase e efeito verde (FAGAN et al., 2010; SOARES et al., 2011; LIMA et al., 2012; PAULO JÚNIOR et al., 2013; HONORATO JÚNIOR et al., 2015); maior qualidade da pós-colheita dos frutos (RAMOS et al., 2013) e aumento da produtividade (KOZLOWSKI et al, 2009; FAGAN et al., 2010; SOARES et al., 2011; DIAZ-ESPEJO et al., 2012).

Os fungicidas de efeitos fisiológicos podem trazer grandes benefícios para a cafeicultura por apresentarem esta dupla ação. Em lavouras cafeeiras, esse efeito levou ao uso dos fungicidas sistêmicos, aplicados via solo, em algumas situações, de forma indiscriminada e com base em outros fins que não ao controle da doença (MARTINS et al., 2011). 
Segundo Zambolim et al. (2009) em médio e/ou longo prazo a aplicação anual desses produtos pode influenciar negativamente na vida útil das lavouras ocasionando baixas produtividades e a insustentabilidade da cultura do café conilon.

Diante do exposto, objetivou-se avaliar o efeito de fungicidas no controle da ferrugem, no crescimento vegetativo e reprodutivo do café conilon.

\section{MATERIAL E MÉTODOS}

O experimento foi conduzido em condições de campo, de julho de 2015 a julho de 2016, no Córrego São José, Chapadão da Onça, Km 04, no município de São Domingos do Norte, noroeste do Estado do Espírito Santo, Brasil (198'40'S; 40³7'28''W; altitude de 108m).

O clima, conforme classificação de Köppen é Aw, tropical com estação seca no inverno e verão chuvoso (ALVARES et al., 2014). As principais variáveis meteorológicas registradas durante a condução do experimento foram: pluviosidade $(\mathrm{mm})$, umidade relativa do ar $(\%)$ e temperatura $\left({ }^{\circ} \mathrm{C}\right)$ (Figura 1).
(3.333 plantas ha-1) e, usando sistema de fertirrigação por gotejo.

$\mathrm{O}$ experimento foi instalado em delineamento em blocos casualizados (DBC) com seis tratamentos (Tabela 1) e quatro repetições, sendo cinco plantas úteis por parcela e quatro plantas como bordadura. As épocas das aplicações foram realizadas de acordo com o calendário utilizado pelos produtores rurais da região (Tabela 1).

A concentração, o grupo químico, a formulação e a dose (produto comercial) dos princípios ativos utilizados estão descritos na Tabela 2.

Todas as pulverizações foram feitas com a adição de óleo mineral (Assist ${ }^{\circledR}$ ) - adjuvante na concentração de $756 \mathrm{~g} \mathrm{~L}^{-1}$, no preparo da calda no momento da pulverização foliar.

As pulverizações com os fungicidas foram realizadas com o pulverizador costal FT-25 YAMAHO ${ }^{\circledR}$ motor tipo elétrico, com capacidade de $25 \mathrm{~L}$, cuja bomba é diafragma, vazão máxima de $4 \mathrm{~L} \mathrm{~min}^{-1}$ e pressão máxima de $0,4 \mathrm{mpa}$, pesando vazio $9,5 \mathrm{~kg}$ e bico duplo. As aplicações foram feitas às sete horas da manhã, com temperatura média inferior a $27^{\circ} \mathrm{C}$, umidade relativa média superior a $75 \%$ e velocidade média do vento de $2 \mathrm{~m} \mathrm{~s}^{-1}$.

A correção e a adubação do solo foram baseadas na análise química do solo, conforme as recomendações para o uso de corretivos e fertilizantes no Estado do Espírito Santo (PREZOTTI et al., 2007).

Os tratos culturais foram realizados de acordo com o recomendado para a cultura. Os dados meteorológicos médios foram obtidos na estação meteorológica convencional, para o município de São Gabriel da Palha - ES, por ser a mais próxima do experimento.

A avaliação do progresso da ferrugem do cafeeiro foi realizada por meio de coletas mensais, quando 10 folhas por planta foram retiradas ao acaso nas quatro posições dos pontos cardeais, no terceiro ou quarto par de folhas dos ramos plagiotrópicos, localizados no terço médio das plantas, perfazendo um total de 50 folhas por parcela (200 folhas por tratamento). Após a coleta das folhas, foi avaliada a incidência da doença, em que dividiu-se o número de folhas com pústulas de ferrugem pelo número total de folhas coletadas e multiplicou-se por 100 para transformar em porcentagem. Com os dados médios de incidência traçou-se as curvas de progresso da doença para cada tratamento (CAPUCHO et al., 2013b).

Para a avaliação do crescimento de ramos, foram marcados aleatoriamente um ramo plagiotrópico e um ramo ortotrópico por planta, com aproximadamente três nós, sendo as medições realizadas com o auxílio de uma fita métrica antes de cada uma das aplicações dos fungicidas (julho/2015, agosto/2015, novembro/2015 e março/2016), juntamente com a contagem do número de nós em cada ramo marcado. O comprimento médio dos internódios foi obtido dividindo-se o comprimento do ramo pelo número de nós de cada ramo.

As avaliações do índice relativo de clorofila (clorofila $a$, clorofila $b$ e clorofila total) foram realizadas com o auxílio de um medidor eletrônico do teor de clorofila (clorofiLOG) portátil CFL 1030 da marca Falker ${ }^{\circledR}$, no terceiro e quarto par de folhas completamente desenvolvidas, selecionadas ao acaso no terço médio das plantas, aos 15 dias após a última aplicação, conforme o exposto por Paulo Júnior et al. (2013). A relação clorofila $a / b$ foi obtida dividindo-se os índices de clorofila $a$ pelos índices de clorofila $b$ em cada folha avaliada. Utilizado o genótipo $02 \mathrm{~V}$, que faz parte da cultivar 'Vitoric INCAPER 8142' (FONSECA et al., 2004). A lavoura fo instalada em agosto de 2013, em espaçamento 3,0 x 1,0 m 
Para as contagens do número médio de rosetas com frutos, frutos por ramo e frutos por roseta, foram escolhidos, aleatoriamente, dois ramos plagiotrópicos (com maior potencial de produção para safra 2015/2016) do terço médio superior de cada planta (décimo ramo), sendo um ramo situado no sentido leste e outro no sentido oeste.

A uniformidade de maturação dos frutos foi avaliada calculando-se os valores médios da porcentagem de frutos verdes, cereja e passa/secos, por meio de contagem em amostras de 300 frutos por parcela, conforme a metodologia proposta por Paiva et al. (2012). Posteriormente, na mesma amostra, foi realizada a pesagem dos frutos por meio de uma balança digital SF-400. Os valores médios da porcentagem de frutos chochos foi avaliada utilizando-se a metodologia proposta por Antunes; Carvalho, (1954), em que 100 frutos maduros foram colocados em água e foram considerados chochos aqueles que permaneceram na superfície.

A avaliação da produtividade do cafeeiro foi realizada por colheita manual dos frutos, nas plantas de cada parcela, no mês de julho de 2016. A produção média dos grãos foi quantificada em litros por planta, e extrapolada para sacas por hectare com a relação de 320 litros igual a uma saca de $60 \mathrm{~kg}$ de café beneficiado, de acordo com o realizado por Oliosi et al. (2016).

A média dos dados obtidos em cada repetição foram submetidas à análise de variância e as médias dos tratamentos foram testadas pelo teste de Tukey a $5 \%$ de probabilidade, através do programa ASSISTAT ${ }^{\circledR}$ Versão 7.7 pt (SILVA; AZEVEDO, 2016).

Tabela 1. Tratamentos e as épocas de aplicação dos fungicidas no cafeeiro conilon. São Domingos do Norte (ES). Table 1. Treatments and the times of application of the fungicides in conilon coffee. São Domingos do Norte (ES)

\begin{tabular}{|c|c|c|c|c|}
\hline \multirow{3}{*}{ Tratamentos } & \multicolumn{4}{|c|}{ Épocas de aplicação } \\
\hline & $1^{\mathrm{a}}$ Aplicação & $2^{\mathrm{a}}$ Aplicação & $3^{\mathrm{a}}$ Aplicação & $4^{\mathrm{a}}$ Aplicação \\
\hline & jul/15 & ago/15 & nov/15 & $\mathrm{mar} / 16$ \\
\hline $\mathrm{T} 1$ & $\begin{array}{c}\text { Piraclostrobina e } \\
\text { Epoxiconazol + Boscalida }\end{array}$ & $\begin{array}{c}\text { Piraclostrobina e } \\
\text { Epoxiconazol + Boscalida }\end{array}$ & $\begin{array}{l}\text { Piraclostrobina e } \\
\text { Epoxiconazol }\end{array}$ & $\begin{array}{l}\text { Piraclostrobina e } \\
\text { Epoxiconazol }\end{array}$ \\
\hline $\mathrm{T} 2$ & $\begin{array}{l}\text { Piraclostrobina e } \\
\text { Epoxiconazol }\end{array}$ & 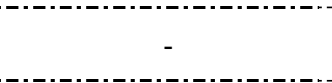 & $\begin{array}{l}\text { Piraclostrobina e } \\
\text { Epoxiconazol }\end{array}$ & $\begin{array}{l}\text { Piraclostrobina e } \\
\text { Epoxiconazol }\end{array}$ \\
\hline $\mathrm{T} 3$ & $\begin{array}{c}\text { Piraclostrobina, Epoxiconazol } \\
\text { e Fluxapiroxade }\end{array}$ & - & $\begin{array}{c}\text { Piraclostrobina, Epoxiconazol } \\
\text { e Fluxapiroxade }\end{array}$ & $\begin{array}{c}\text { Piraclostrobina, Epoxiconazol } \\
\text { e Fluxapiroxade }\end{array}$ \\
\hline $\mathrm{T} 4$ & Epoxiconazol & - & Epoxiconazol & Epoxiconazol \\
\hline T5 & Piraclostrobina & - & Piraclostrobina & Piraclostrobina \\
\hline $\mathrm{T} 6$ & Testemunha & Testemunha & Testemunha & Testemunha \\
\hline
\end{tabular}

Tabela 2. Princípio ativo, grupo químico, formulação e dose (g ou L $\mathrm{ha}^{-1}$ ) dos fungicidas aplicados no cafeeiro conilon. São Domingos do Norte (ES).

Table 2. Active principle, chemical group, formulation and dose ( $\mathrm{g}$ or $\mathrm{L} \mathrm{ha}^{-1}$ ) of the fungicides applied in conilon coffee. São Domingos do Norte (ES).

\begin{tabular}{|c|c|c|c|}
\hline Princípio ativo $^{1}$ & $\begin{array}{c}\text { Grupo } \\
\text { Químico }\end{array}$ & $\begin{array}{l}\text { Dose } \\
(\text { p.c. })^{2}\end{array}$ & Formulaçãa ${ }^{3}$ \\
\hline $\begin{array}{c}\text { Piraclostrobina } \\
133,0 \mathrm{~g} \mathrm{~L}^{-1} \mathrm{e} \\
\text { Epoxiconazol } \\
50,0 \mathrm{~g} \mathrm{~L}^{-1}\end{array}$ & $\begin{array}{c}\text { Estrobilurina e } \\
\text { Triazol }\end{array}$ & $\begin{array}{c}1,5 \\
\mathrm{~L} \mathrm{ha-1}\end{array}$ & SE \\
\hline $\begin{array}{l}\text { Boscalida } \\
500 \mathrm{~g} \mathrm{~kg}^{-1}\end{array}$ & Anilida & $\begin{array}{l}150 \\
\mathrm{~g} \mathrm{ha}^{-1}\end{array}$ & WG \\
\hline $\begin{array}{l}\text { Piraclostrobina } \\
\text { 64,8 } \mathrm{g} \mathrm{L}^{-1} \\
\text { Epoxiconazol } \\
40,0 \mathrm{~g} \mathrm{~L}^{-1} \mathrm{e} \\
\text { Fluxapiroxade } \\
40,0 \mathrm{~g} \mathrm{~L}^{-1}\end{array}$ & $\begin{array}{l}\text { Estrobilurina, } \\
\text { Triazol e } \\
\text { Carboxamida }\end{array}$ & $\begin{array}{c}1,5 \\
\mathrm{~L} \mathrm{ha}^{-1}\end{array}$ & $\mathrm{EC}$ \\
\hline $\begin{array}{c}\text { Epoxiconazol } \\
125,0 \mathrm{~g} \mathrm{~L}^{-1}\end{array}$ & Triazol & $\begin{array}{c}0,6 \\
\mathrm{~L} \mathrm{ha}^{-1}\end{array}$ & $\mathrm{SC}$ \\
\hline $\begin{array}{l}\text { Piraclostrobina } \\
250,0 \mathrm{~g} \mathrm{~L}^{-1}\end{array}$ & Estrobilurina & $\begin{array}{c}0,6 \\
\mathrm{~L} \mathrm{ha}^{-1}\end{array}$ & $\mathrm{EC}$ \\
\hline
\end{tabular}

Legenda: ${ }^{1}$ AGROFIT (2017); ${ }^{2}$ produto comercial; ${ }^{3}$ SE: suspo/emulsão; WG: granulado dispersível; EC: concentrado emulsionável; SC: suspensão concentrada.

\section{RESULTADOS}

Durante o período estudado, a precipitação acumulada foi $582,2 \mathrm{~mm}, 51,5 \%$ abaixo da média esperada para o período e a umidade relativa do ar foi em média $75,4 \%$. Em relação à temperatura do ar, os valores médios e mínimos foram $25,2^{\circ} \mathrm{C}$ e $19,7^{\circ} \mathrm{C}$, respectivamente, enquanto os valores máximos foram em média $32,8^{\circ} \mathrm{C}$ (Figura 1). Durante as avaliações, não houve ocorrência da ferrugem do cafeeiro nas plantas. Assim, não foi possível traçar as curvas de progresso da doença.

Em relação ao crescimento acumulado (Tabela 3) e número de nós (Tabela 4) houve diferença apenas para os ramos plagiotrópicos, na segunda avaliação (novembro/2015). No entanto, o comprimento médio dos internódios foram semelhantes em todos os tratamentos (Tabela 5). Nesta avaliação, a aplicação somente com epoxiconazol (T4) proporcionou uma redução de $25,8 \%$ e $10,4 \%$, para o crescimento acumulado e número de nós por ramo, respectivamente.

Os valores médios dos índices de clorofila $a$, clorofila $b$ e clorofila total, nas folhas do cafeeiro conilon, variaram em função dos tratamentos. Os maiores valores foram obtidos com a aplicação de piraclostrobina (T5); piraclostrobina, epoxiconazol e fluxapiroxade (T3); piraclostrobina e epoxiconazol + boscalida (T1); piraclostrobina e epoxiconazol (T2) (Tabela 6).

Quanto a relação clorofila $a / b$ observou-se que a mesma foi reduzida, nas plantas pulverizadas com piraclostrobina (T5) e piraclostrobina, epoxiconazol e fluxapiroxade (T3). A aplicação de piraclostrobina e epoxiconazol + boscalida (T1) e piraclostrobina e epoxiconazol (T2) proporcionaram valores intermediários da relação clorofila $a / b$ (Tabela 6 ). O número de frutos por ramo e o número de frutos por roseta não foram influenciados pelos diferentes tratamentos (Tabela 6).

Em relação ao número de rosetas com frutos houve diferença entre os tratamentos, com valores superiores para a testemunha (T6), a aplicação de piraclostrobina, epoxiconazol 
e fluxapiroxade (T3), piraclostrobina e epoxiconazol + boscalida (T1), piraclostrobina (T5) e piraclostrobina e epoxiconazol (T2) (Tabela 6). A aplicação isolada de epoxiconazol (T4) proporcionou uma redução de $10,6 \%$ nesta variável.

De acordo com a Tabela 7, houve a presença de frutos chochos, no entanto, não foi influenciada significativamente com a aplicação dos diferentes fungicidas. Quanto a uniformidade de maturação dos frutos não houve variação nos resultados de porcentagem de frutos verdes, cereja e passa/secos com a aplicação dos tratamentos (Tabela 7). Observou-se também, que nenhum produto pulverizado alterou o peso dos frutos colhidos nas respectivas plantas tratadas. Os resultados de produtividade variaram entre 69,3 a 90,1 sacas ha ${ }^{-1}$, entretanto não diferenciaram entre si.

\section{DISCUSSÃO}

Durante o período estudado, a precipitação acumulada foi $582,2 \mathrm{~mm}, 51,5 \%$ abaixo da média esperada para o período e a umidade relativa do ar foi em média $75,4 \%$. Em relação à temperatura do ar, os valores médios e mínimos foram $25,2^{\circ} \mathrm{C}$ e $19,7^{\circ} \mathrm{C}$, respectivamente, enquanto os valores máximos foram em média $32,8^{\circ} \mathrm{C}$ (Figura 1 ).

Para o café conilon, as condições ambientais favoráveis para a infecção da $H$. vastatrix são temperaturas médias moderadas entre 21,6 a $23,6^{\circ} \mathrm{C}$ com umidade foliar associado à umidade relativa (maior que 80\%) (CAPUCHO et al., 2013a). Isso justifica a não ocorrência da ferrugem do cafeeiro durante as avalições.

Quanto ao crescimento das plantas, resultados semelhantes foram observados por Martins et al. (2011). Os autores observaram a redução do crescimento das plantas de café conilon, cultivar 'Vitória Incaper 8142 ' e, além disso, o aparecimento de sintomas de fitotoxidez com a aplicação de ciproconazol (triazol) + tiametoxam (neonicotinóide) via solo.

$\mathrm{Na}$ cultura do trigo, o maior efeito no desenvolvimento foi observado quando a piraclostrobina foi aplicada durante a fase em que a demanda de nitrogênio é máxima (KÖHLE et al., 2003; VENÂNCIO et al., 2003). A maior demanda do nutriente, na cultura do café conilon, ocorre durante o enfolhamento, iniciando-se após a colheita, no mês de julho (PREZOTTI et al., 2015), período o qual foi realizada a primeira pulverização dos tratamentos, sugerindo o comportamento do maior crescimento das plantas pulverizadas com estrobilurina na segunda avaliação (novembro/2015).

As médias superiores nas plantas tratadas com a piraclostrobina, na avaliação de novembro/2015, permite associar a aplicação da molécula com o maior desenvolvimento vegetativo, mesmo em condições de temperatura elevadas e limitação de água, comparado ao tratamento com epoxiconazol.

O maior número de nós desenvolvidos no ramo do ano das plantas submetidas aos tratamentos com piraclostrobina (Tabela 4), demonstra um efeito no alongamento celular nos tecidos jovens da região, aumentando, consequentemente, o seu comprimento. Isso é fundamental para garantir a produção no ano seguinte, que depende dos mesmos, pois serão neles que estarão localizados os frutos e também as folhas que serão consideradas como fonte (COSTA et al., 2009) de fotoassimilados direcionados ao enchimento dos frutos.

Tabela 3. Crescimento acumulado de ramos plagiotrópicos e ortotrópicos, do cafeeiro conilon, em resposta à aplicação dos tratamentos. Table 3. Cumulative growth of plagiotropic and orthotropic branches of conilon coffee in response to treatments.

\begin{tabular}{|c|c|c|c|c|c|c|c|c|}
\hline \multirow{3}{*}{ Trat. } & \multicolumn{8}{|c|}{ Crescimento de ramos } \\
\hline & \multicolumn{4}{|c|}{ Ramos Plagiotrópicos (cm) } & \multicolumn{4}{|c|}{ Ramos Ortotrópicos (cm) } \\
\hline & $\mathrm{Jul} / 15$ & Nov/15 & Mar/16 & $\mathrm{Jul} / 16$ & Jul/15 & Nov/15 & Mar/16 & Jul/16 \\
\hline T1 & 9,3 & $11,8 \mathrm{ab}$ & 32,2 & 36,4 & 5,6 & 9,4 & 26,2 & 29,5 \\
\hline $\mathrm{T} 2$ & 8,8 & $12,9 \mathrm{ab}$ & 33,3 & 37,3 & 5,5 & 9,9 & 27,2 & 30,4 \\
\hline T3 & 8,6 & $10,3 \mathrm{ab}$ & 31,0 & 35 & 4,9 & 8,9 & 25,2 & 29,3 \\
\hline T4 & 8,4 & $9,10 \mathrm{~b}$ & 31,0 & 34,9 & 5,1 & 8,1 & 25,6 & 29,6 \\
\hline T5 & 8,1 & $14,3 \mathrm{a}$ & 35,4 & 40,1 & 4,8 & 11,3 & 29,7 & 34,5 \\
\hline T6 & 8,3 & $12,0 \mathrm{ab}$ & 31,8 & 34,8 & 5,9 & 9,0 & 25,4 & 29,3 \\
\hline $\mathrm{CV}^{1}$ & 11,7 & 18,5 & 10,6 & 12,2 & 17,7 & 17,7 & 11,5 & 12,1 \\
\hline $\mathrm{F}^{2}$ & $0,70^{\mathrm{ns}}$ & $2,87^{\mathrm{ns}}$ & $0,98^{\mathrm{ns}}$ & $0,85^{\mathrm{ns}}$ & $0,82^{\text {ns }}$ & $1,65^{\mathrm{ns}}$ & $1,27^{\mathrm{ns}}$ & $1,21^{\mathrm{ns}}$ \\
\hline $\mathrm{dms}^{3}$ & 2,3 & 5,0 & 7,9 & 10,2 & 2,1 & 3,8 & 7,0 & 8,4 \\
\hline
\end{tabular}

Médias seguidas por uma mesma letra, em cada coluna, não diferem estatisticamente (Tukey, $\mathrm{p}>0,05) .{ }^{1}$ Coeficiente de variação em $\%{ }^{2}$ Estatística do teste $\mathrm{F}$. ${ }^{3}$ Diferença mínima significativa.

Tabela 4. Número de nós por ramo plagiotrópico e ortotrópico, no cafeeiro conilon, em resposta à aplicação dos tratamentos.

Table 4. Number of nodes per plagiotropic and orthotropic branch in the conilon coffee tree, in response to the application of the treatments.

\begin{tabular}{ccccccccc}
\hline & \multicolumn{9}{c}{ Número de nós } \\
\cline { 2 - 9 } Trat. & \multicolumn{3}{c}{ Ramos Plagiotrópicos (cm) } & \multicolumn{3}{c}{ Ramos Ortotrópicos (cm) } \\
\cline { 2 - 9 } & Jul/15 & Nov/15 & Mar/16 & Jul/16 & Jul/15 & Nov/15 & Mar/16 & Jul/16 \\
\hline T1 & 2,5 & $7,5 \mathrm{ab}$ & 14,2 & 16,6 & 2,7 & 6,7 & 13,2 & 15,6 \\
T2 & 2,5 & $7,5 \mathrm{ab}$ & 14,4 & 16,7 & 2,5 & 6,8 & 13,5 & 15,4 \\
T3 & 2,3 & $7,1 \mathrm{ab}$ & 13,9 & 16,3 & 2,3 & 6,4 & 12,5 & 15,2 \\
T4 & 2,3 & $6,7 \mathrm{~b}$ & 13,4 & 16,0 & 2,4 & 6,1 & 12,2 & 14,8 \\
T5 & 2,2 & $7,8 \mathrm{a}$ & 14,9 & 16,7 & 2,3 & 6,9 & 13,7 & 16,4 \\
T6 & 2,5 & $7,5 \mathrm{ab}$ & 13,8 & 15,7 & 2,7 & 6,6 & 12,8 & 14,8 \\
\hline $\mathrm{CV}^{1}$ & 11,8 & 6,4 & 6,4 & 8,1 & 12,9 & 7,5 & 6,9 & 6,5 \\
$\mathrm{~F}^{2}$ & $0,78^{\text {ns }}$ & $2,64^{\text {ns }}$ & $1,28^{\text {ns }}$ & $0,41^{\text {ns }}$ & $1,37^{\text {ns }}$ & $1,36^{\text {ns }}$ & $1,78^{\text {ns }}$ & $1,46^{\text {ns }}$ \\
dms $^{3}$ & 0,6 & 1,1 & 2,1 & 3,0 & 0,7 & 1,1 & 2,1 & 2,3 \\
\hline
\end{tabular}

Médias seguidas por uma mesma letra, em cada coluna, não diferem estatisticamente (Tukey, p>0,05). ${ }^{1}$ Coeficiente de variação em $\%$. ${ }^{2}$ Estatística do teste F. ${ }^{3}$ Diferença mínima significativa. 
Tabela 5. Comprimento médio de internódios de ramos plagiotrópicos e ortotrópicos, no cafeeiro conilon, em resposta à aplicação dos tratamentos.

Table 5. Mean length of internodes of plagiotropic and orthotropic branches in conilon coffee, in response to treatments.

\begin{tabular}{ccccccccc}
\hline & \multicolumn{9}{c}{ Comprimento de internódios } \\
\cline { 2 - 9 } Trat. & \multicolumn{3}{c}{ Ramos Plagiotrópicos (cm) } & \multicolumn{3}{c}{ Ramos Ortotrópicos (cm) } \\
\cline { 2 - 8 } & Jul/15 & Nov/15 & Mar/16 & Ju1/16 & Jul/15 & Nov/15 & Mar/16 & Ju1/16 \\
\hline T1 & 3,8 & 2,8 & 2,9 & 2,8 & 2,1 & 2,3 & 2,4 & 2,3 \\
T2 & 3,6 & 2,9 & 2,9 & 2,8 & 2,2 & 2,3 & 2,4 & 2,3 \\
T3 & 3,8 & 2,7 & 2,8 & 2,7 & 2,2 & 2,2 & 2,4 & 2,2 \\
T4 & 3,7 & 2,6 & 2,9 & 2,7 & 2,2 & 2,2 & 2,5 & 2,4 \\
T5 & 3,8 & 2,9 & 2,9 & 2,9 & 2,1 & 2,3 & 2,5 & 2,4 \\
T6 & 3,4 & 2,7 & 2,9 & 2,7 & 2,2 & 2,3 & 2,5 & 2,4 \\
\hline CV $^{1}$ & 9,4 & 9,1 & 5,8 & 5,5 & 9,5 & 8,9 & 7,5 & 7,2 \\
F $^{2}$ & $0,87^{\text {ns }}$ & $0,81^{\text {ns }}$ & $0,13^{*}$ & $0,81^{\text {ns }}$ & $0,22^{\text {ns }}$ & $0,34^{\text {ns }}$ & $0,36^{\text {ns }}$ & $0,52^{\text {ns }}$ \\
dms $^{3}$ & 0,8 & 0,6 & 0,4 & 0,4 & 0,5 & 0,5 & 0,4 & 0,4 \\
\hline
\end{tabular}

Médias seguidas por uma mesma letra, em cada coluna, não diferem estatisticamente (Tukey, p $>0,05$ ). ${ }^{1}$ Coeficiente de variação em $\% .{ }^{2}$ Estatística do teste F. ${ }^{3}$ Diferença mínima significativa.

Tabela 6. Valores médios dos índices de clorofila $a$, clorofila $b$, clorofila total e relação clorofila $a / b$, do número de frutos por ramo, número de rosetas com frutos e do número de frutos por roseta, no cafeeiro conilon, em resposta à aplicação dos tratamentos, após a última aplicação. Table 6. Mean values of chlorophyll $a$, chlorophyll $b$, total chlorophyll and chlorophyll $a / b$, number of fruits per branch, number of rosettes with fruits and number of fruits per rosette, in conilon coffee, in response to after the last application.

\begin{tabular}{cccccccc}
\hline Trat. & $\begin{array}{c}\text { Clorofila } a \\
\text { (ICF) }\end{array}$ & $\begin{array}{c}\text { Clorofila } b \\
\text { (ICF) }\end{array}$ & $\begin{array}{c}\text { Clorofila Total } \\
\text { (ICF) }\end{array}$ & $\begin{array}{c}\text { Relação Clorofila } \\
a / b\end{array}$ & $\begin{array}{c}\text { Número de } \\
\text { frutos por } \\
\text { ramo }\end{array}$ & $\begin{array}{c}\text { Número de } \\
\text { rosetas com } \\
\text { frutos }\end{array}$ & $\begin{array}{c}\text { Número de } \\
\text { frutos por } \\
\text { roseta }\end{array}$ \\
\hline $\mathrm{T} 1$ & $39,8 \mathrm{abc}$ & $22,2 \mathrm{ab}$ & $62,0 \mathrm{abc}$ & $1,81 \mathrm{bc}$ & 80,5 & $16,8 \mathrm{ab}$ & 4,8 \\
$\mathrm{~T} 2$ & $39,4 \mathrm{abc}$ & $21,8 \mathrm{abc}$ & $61,1 \mathrm{abc}$ & $1,81 \mathrm{bc}$ & 96,2 & $16,5 \mathrm{ab}$ & 6,0 \\
$\mathrm{~T} 3$ & $40,3 \mathrm{ab}$ & $23,1 \mathrm{ab}$ & $63,5 \mathrm{ab}$ & $1,74 \mathrm{c}$ & 95,1 & $16,9 \mathrm{ab}$ & 5,7 \\
$\mathrm{~T} 4$ & $38,0 \mathrm{bc}$ & $17,8 \mathrm{bc}$ & $55,7 \mathrm{bc}$ & $2,17 \mathrm{ab}$ & 74,6 & $15,1 \mathrm{~b}$ & 5,0 \\
$\mathrm{~T} 5$ & $40,6 \mathrm{a}$ & $24,0 \mathrm{a}$ & $64,6 \mathrm{a}$ & $1,70 \mathrm{c}$ & 81,2 & $16,7 \mathrm{ab}$ & 4,9 \\
$\mathrm{~T} 6$ & $37,4 \mathrm{c}$ & $16,3 \mathrm{c}$ & $53,8 \mathrm{c}$ & $2,29 \mathrm{a}$ & 95,2 & $17,6 \mathrm{a}$ & 5,4 \\
\hline $\mathrm{CV}^{1}$ & 2,9 & 12,1 & 6,0 & 9,39 & 17,5 & 5,8 & 19,5 \\
$\mathrm{~F}^{2}$ & $5,12^{* *}$ & $5,97^{* *}$ & $5,87^{* *}$ & $7,50^{* *}$ & $1,53^{\mathrm{ns}}$ & $2,93^{*}$ & $0,87^{\mathrm{ns}}$ \\
$\mathrm{dms}^{3}$ & 2,6 & 5,8 & 8,3 & 0,41 & 34,9 & 2,2 & 2,4
\end{tabular}

Médias seguidas por uma mesma letra, em cada coluna, não diferem estatisticamente (Tukey, $\mathrm{p}>0,05) .{ }^{1}$ Coeficiente de variação em $\%{ }^{2}$ Estatística do teste $\mathrm{F}$. ${ }^{3}$ Diferença mínima significativa.

Tabela 7. Valores médios de porcentagem de frutos chochos, porcentagem de frutos verdes, porcentagem de frutos cereja e porcentagem de frutos passa/secos, do peso dos frutos e produtividade, do cafeeiro conilon, em resposta à aplicação dos tratamentos.

Table 7. Mean percentage values of chochos fruits, percentage of green fruits, percentage of cherry fruits and percentage of nuts / dry fruits, fruit weight and yield of conilon coffee, in response to treatments.

\begin{tabular}{|c|c|c|c|c|c|c|}
\hline \multirow{2}{*}{ Trat. } & \multicolumn{4}{|c|}{ Porcentagem média de frutos } & \multirow[b]{2}{*}{ Peso dos frutos $(\mathrm{g})$} & \multirow[b]{2}{*}{ Produtividade ${ }^{4}$} \\
\hline & Chochos $(\%)$ & Verdes $(\%)$ & Cereja (\%) & Passa/Secos (\%) & & \\
\hline T1 & 6,7 & 41,1 & 54,1 & 4,8 & 256,5 & 86,7 \\
\hline $\mathrm{T} 2$ & 9,3 & 32,5 & 60,8 & 6,8 & 260,5 & 87,5 \\
\hline T3 & 10,0 & 36,1 & 58,1 & 5,8 & 249,5 & 69,3 \\
\hline T4 & 9,7 & 28,4 & 67,1 & 4,5 & 255,8 & 75,5 \\
\hline T5 & 6,2 & 30,2 & 65,6 & 4,2 & 245,3 & 90,1 \\
\hline $\mathrm{T} 6$ & 9,2 & 43,7 & 51,0 & 5,3 & 243,0 & 80,2 \\
\hline $\mathrm{CV}^{1}$ & 25,5 & 31,2 & 17,4 & 24,7 & 7,1 & 20,4 \\
\hline $\mathrm{F}^{2}$ & $2,26^{\mathrm{ns}}$ & $1,22^{\mathrm{ns}}$ & $1,50^{\mathrm{ns}}$ & $2,08^{\mathrm{ns}}$ & $0,54^{\mathrm{ns}}$ & $0,93^{\mathrm{ns}}$ \\
\hline $\mathrm{dms}^{3}$ & 5,0 & 25,3 & 23,7 & 3,0 & 41,0 & 38,1 \\
\hline
\end{tabular}

Médias seguidas por uma mesma letra, em cada coluna, não diferem estatisticamente (Tukey, $\mathrm{p}>0,05) .{ }^{1}$ Coeficiente de variação em $\% .{ }^{2}$ Estatística do teste $\mathrm{F}$. Diferença mínima significativa.

O tratamento somente com epoxiconazol proporcionou a redução do crescimento acumulado e do número de nós por ramo na avaliação de novembro/2015. Segundo Soares et al. (2005), a redução do crescimento realmente induz a uma menor produção de nós disponíveis para a formação de flores, o que ocasiona, na queda na produção de frutos. No entanto, observou-se que as plantas tratadas com epoxiconazol não apresentaram sintomas de fitotoxidez, como internódios curtos, devido aos valores do comprimento médio dos internódios semelhantes aos demais tratamentos em todas as avaliações (Tabela 5). Experimentos realizados por Martins et al. (2011) com a aplicação de triazol (ciproconazol) + neonicotinóide (tiametoxam) no café conilon evidenciaram vários sintomas de fitotoxidez, dentre eles internódios curtos.

Ressalta-se que em todos os tratamentos em que houve o aumento nos valores dos índices de clorofila contam com presença da estrobilurina (piraclostrobina) (Tabela 6). Nos frutos de tomate 'Giuliana' os teores de clorofila $a$ e $b$ foram influenciados com aplicações de boscalida, piraclostrobina e mistura de piraclostrobina + boscalida (RAMOS et al., 2013). Em mudas de bananeira, os teores de clorofila $a$ também foram superiores nas plantas tratadas com piraclostrobina em relação às tratadas com azoxystrobina e água. Entretanto, os conteúdos 
de clorofila $b$ não foram alterados com a aplicação das estrobilurinas (LIMA et al., 2012).

Estudo sobre as alterações fotossintéticas e antioxidantes nas folhas de café arábica, causadas pelo uso de fungicidas e a infecção de Hemileia vastatrix, foi verificado que o uso dos fungicidas epoxiconazol e piraclostrobina ao mesmo tempo em que restringiram o desenvolvimento da doença, promoveram maior concentração de clorofilas nas plantas (HONORATO JÚNIOR et al., 2015). Na mesma variedade de café arábica, Paulo Júnior et al. (2013), observaram que em plantas tratadas com piraclostrobina, houve aumento da intensidade da cor verde (índice SPAD), levando ao aumento nos teores de clorofila total. Diferente dos resultados obtidos neste trabalho, nas folhas do feijoeiro não foi evidenciado o efeito das estrobilurinas (piraclostrobina, azoxystrobina e trifloxistrobina) no aumento da clorofila (KOZLOWSKI et al., 2009).

A pulverização com piraclostrobina, em mudas de bananeira, proporcionou uma maior atividade da nitrato redutase e, também valores mais altos de aminoácidos livres totais e de nitrogênio total, indicando uma maior assimilação de nitrogênio (LIMA et al., 2012). Segundo ao autores, tal fato foi justificado pelos teores mais altos de compostos nitrogenados relacionados com a assimilação de carbono, tais como pigmentos fotossintéticos (clorofilas), o que resulta em maior crescimento das plantas, como observado na Tabela 3.

A nitrato redutase também apresenta uma rota alternativa que produz óxido nítrico, que inibe as enzimas chaves da biossíntese do etileno, ACC sintase e ACC oxidase (VENÂNCIO et al., 2003). A redução dos níveis de etileno, diminuem a degradação das citocininas, aumentando a persistência da clorofila, resultando o que é chamado de "efeito verde" (GROSSMANN \& RETZLAFF, 1997). Assim, as estrobilurinas induzem a síntese da clorofila, fenômeno conhecido como "efeito verde", que está associado com o atraso da senescência e a maximização do rendimento (HONORATO JÚNIOR et al., 2015).

Quanto a relação clorofila $a / b$ observou-se que os resultados foram semelhantes aos relatados por Honorato Júnior et al. (2015), que encontraram a relação clorofila $a / b$ reduzida nas folhas do cafeeiro inoculadas e pulverizadas com piraclostrobina aos 2 e aos 10 dias após a inoculação.

A aplicação isolada de epoxiconazol reduziu o número de rosetas com frutos, indicando uma menor produtividade.

Avaliação do efeito fitotônico de diferentes princípios ativos, dentre os quais a piraclostrobina e carboxamida, aplicados via tratamento de sementes, não resultou no aumento do número de vagens por planta na cultura da soja (CUNHA et al., 2015), dados contraditórios aos obtidos neste estudo.

No feijoeiro, o número de grãos por vagem também não foram influenciados com a aplicação de diferentes fungicidas (KOZLOWSKI et al., 2009). Na mesma cultura, Demant; Maringoni (2012), observaram que os fungicidas testados não provocaram grandes variações do número de vagens por planta e número de grãos por vagem em condições controladas.

Quando a cultura do cafeeiro é afetada por agentes bióticos, como as doenças mancha-de-phoma (Phoma costaricencis) e mancha-de-ascochyta (Ascochyta coffeae), o tratamento de florada com fungicida boscalida resulta um maior controle, e consequentemente, menor perda de produtividade. $\mathrm{O}$ fungicida quando pulverizado preventivamente e no momento que antecede $o$ desenvolvimento da doença, reduz o índice de infecção do complexo phoma/ascochyta, aumentando o número de frutos por roseta e resultando em uma maior produtividade (BASF, 2016).

Não houve diferença nas porcentagens de frutos verdes, cereja e passa/secos, o que mostra que os fungicidas testados não influenciaram na maturação dos frutos. Mesmo com a ocorrência de um período de baixa precipitação durante a condução do experimento (Figura 1), a faixa de produtividade foi superior à média das lavouras de café conilon do Estado que, segundo a CONAB (2017), é 19,3 sacas ha-1.

Segundo Venâncio et al. (2003), mesmo em plantas não infectadas por fungos, é interessante para as práticas agrícolas o aumento da biomassa e da produção obtidos pela aplicação, por exemplo, da piraclostrobina. Entretanto, a aplicação dos fungicidas não influenciaram de forma significativa na produtividade do cafeeiro (Tabela 8).

Os resultados obtidos assemelham-se aos de Demant e Maringoni (2012) em que, nos dois ensaios conduzidos sob condições de telado, não detectaram diferença na produção por planta, com aplicação de azoxistrobina, metiram + piraclostrobina, piraclostrobina, trifloxistrobina, tebuconazole, hidróxido de fentina e tebuconazole + trifloxistrobina no feijão. Aplicação de diferentes princípios ativos, como piraclostrobina e carboxamida, via tratamento de sementes, também não proporcionaram diferença estatística na produção de grãos em plantas de soja (CUNHA et al., 2015). $\mathrm{Na}$ cultura da soja, a aplicação de piraclostrobina (FAGAN et al., 2010), piraclostrobina + epoxiconazole (SOARES et al., 2011) também proporcionaram incremento na produtividade.

A ausência da ferrugem no experimento foi importante para as avaliações realizadas, visto que esta doença pode reduzir a produtividade da cultura (CAPUCHO et al., 2013b), por restringir a área de tecido para a fotossíntese e inibir a translocação de fotoassimilados, desde sua fonte de produção até as áreas de crescimento e deposição de materiais de rendimento, como grãos, frutos, dentre outros (VENÂNCIO et al., 2003).

Nota-se que para uma melhor eficácia dos tratamentos, seria necessária a aplicação dos fungicidas por um tempo maior e, que se fosse avaliar apenas o efeito dos produtos isoladamente, uma avaliação seria ideal.

Mas, verificou-se alguns resultados positivos dos produtos nas avaliações realizadas. Isso mostra que, se em condições de déficit hídrico, como ocorreram durante o experimento, houve algumas diferenças, em condições ideais de temperatura e precipitação os resultados tendem a se destacarem mais.

Ressalta-se que o uso de produtos químicos deve ser recomendado prioritariamente para o controle de doenças e não para o aumento da produtividade. Esse aumento, caso aconteça deve vir em consequência do efeito do produto sobre as atividades do patógeno na planta. Nesse caso, se o produto potencializar outros processos fisiológicos da planta, deve ser entendido como um fator adicional dentro de um programa estruturado de manejo ([Antonio Fernando de Souza], [IFES], comunicação pessoal).

\section{CONCLUSÕES}

A aplicação com epoxiconazol proporcionou menor crescimento acumulado e número de nós por ramo plagiotrópico, apenas na segunda avaliação (novembro/2015) e, menor número de rosetas com frutos. Os tratamentos com piraclostrobina influenciaram positivamente os índices de 
clorofila $a, b$ e total e proporcionaram valores inferiores da relação clorofila $a / b$.

Não houve diferença entre os tratamentos no controle da ferrugem devido as condições adversas para a doença.

\section{AGRADECIMENTOS}

Ao Programa de Pós-Graduação em Agricultura Tropical (PPGAT), ao Conselho Nacional de Desenvolvimento Científico Tecnológico $(\mathrm{CNPq})$, pelo apoio financeiro e a Cooperativa Agrária dos Cafeicultores de São Gabriel (COOABRIEL) pela disponibilização da área para a realização do experimento.

\section{REFERÊNCIAS}

ALVARES, C. A.; STAPE, J. L.; SENTELHAS, P. C.; GONÇALVES, J. L. M.; SPAROVEK, G. Koppen's climate classification map for Brazil. Meteorologische Zeitschrift, v. 22, n. 6, p. 711-728, 2014. DOI: https://doi.org/10.1127/0941-2948/2013/0507

ANTUNES FILHO, H.; CARVALHO, A. Melhoramento do cafeeiro, ocorrência de lojas vazias em frutos de café Mundo Novo. Bragantia, Campinas, v. 14, p. 165-179, 1954. DOI: http://dx.doi.org/10.1590/S000687051954000100014

BASF. Mancha-de-phoma e mancha-de-ascochyta em café. Cooabriel, ano XXX, n. 208, p.7, ago./set. 2016. Disponível em: http://cooabriel.coop.br/wpcontent/uploads/2016/09/COOABRIEL-208-BAIXA.pdf. Acesso em: 16 out. 2016.

CAPUCHO, A. S.; ZAMBOLIM, L.; CABRAL, P. G. C.; MACIEL-ZAMBOLIM, E.; CAIXETA, E. T. Climate favourability to leaf rust in Conilon coffee. Australasian Plant Pathology, v. 42, n. 1, p. 511-514, 2013a. DOI: https://doi.org/10.1007/s13313-012-0187-6

CAPUCHO, A. S.; ZAMBOLIM, L.; LOPES, U. N.; MILAGRES, N.S. Chemical control of coffee leaf rust in Coffea canephora cv. Conilon. Australasian Plant Pathology, v. 42, n. 1, p. 642-667, 2013b. DOI: https://doi.org/10.1007/s13313-013-0242-y

COMPANHIA NACIONAL DE ABASTECIMENTO (CONAB). Acompanhamento da safra brasileira café, V.4 - safra 2017 - N.1 - primeiro levantamento, janeiro 2017. Brasília, 2017. 98p. Disponível em: http://www.conab.gov.br/OlalaCMS/uploads/arquivos/17 01_17_14_51_54_boletim_cafe_-janeiro_de_2017.pdf. Acesso em: 11 mar. 2017.

COSTA, N. R.; DOMINGUES, M. C. S.; RODRIGUES, J. D.; TEIXEIRA FILHO, M. C. M. Desempenho do cafeeiro Icatu vermelho sob ação de biorregulador aplicado em fases reprodutivas da cultura. Agrarian, Dourados, v. 2, n. 5, p. 113-130, 2009.

CUNHA, R. P. da; CORREAA, M. F.; SCHUCH, L. O. B.; OLIVEIRA, R. C. de; ABREU JÚNIOR, J. S. de; SILVA, J. D. G. da; ALMEIDA, T. L. de. Diferentes tratamentos de sementes sobre o desenvolvimento de plantas de soja. Ciência Rural, Santa Maria, v. 45, n. 10, p. 1761-1767, 2015. DOI: http://dx.doi.org/10.1590/0103$8478 \mathrm{cr} 20140742$

DEMANT, L. A. D.; MARINGONI, A. C. Controle da mancha angular do feijoeiro com uso de fungicidas e seu efeito na produção das plantas. Idesia (Arica), v. 30, n. 2, p. 93-100, 2012. DOI: http://dx.doi.org/10.4067/S071834292012000200012

DIAZ-ESPEJO, A.; CUEVAS, M. V.; RIBAS-CARBO, M.; FLEXAS, J.; MARTORELL, S.; FENÁNDES, J. E. The effect of strobilurins on leaf gas exchange, water use efficiency and ABA content in grapevine under field conditions. Jornal of Plant Physiology, v. 169, n. 1, p. 379-386, 2012.

DOI: https://doi.org/10.1016/j.jplph.2011.11.014

FAGAN, E. B.; DOURADO NETO, D.; VIVIAN, R.; FRANCO, R. B.; YEDA, M. P.; MASSIGNAM, L. F.; OLIVEIRA, R. F. de; MARTINS, K. V. Efeito da aplicação de piraclostrobina na taxa fotossintética, respiração, atividade da enzima nitrato redutase e produtividade de grãos de soja. Bragantia, Campinas, v. 69, p. $\quad 771-777, \quad 2010 . \quad$ DOI: http://dx.doi.org/10.1590/S0006-87052010000400001

FONSECA, A. F. A. da; FERRÃO, M. A. G.; FERRÃO, R. G.; VERDIN FILHO, A. C.; VOLPI, P. S.; ZUCATELI, F. 'Conilon Vitória - Incaper 8142': improved Coffea canephora var. Kouillou clone cultivar for the state of Espírito Santo. Crop Breeding and Applied Biotechnology, Viçosa, v. 4, n. 1, p. 503-505, 2004. DOI: https://doi.org/10.12702/1984-7033.v04n04a20

GROSSMANN, K.; RETZLAFF, G. Bioregulatory effects of the fungicidal strobirulin kresoxim-methyl in wheat (Triticum aestivum). Pesticide Science, v. 50, n. 1, p. 1120, 1997. DOI: https://doi.org/10.1002/(SICI)10969063(199705)50:1<11::AID-PS556>3.0.CO;2-8

HONORATO JÚNIOR, J.; ZAMBOLIM, L.; AUCIQUEPÉREZ, C. E.; RESENDE, F. A.; RODRIGUES, F. A. Photosynthetic and antioxidative alterations in coffee leaves caused by epoxiconazole and pyraclostrobin sprays and Hemileia vastatrix infection. Pesticide Biochemistry and Physiology, v. 123, n. 1, p. 31-39, 2015. DOI: https://doi.org/10.1016/j.pestbp.2015.01.016

KÖHLE, H.; GROSSMANN, K.; JABS, T.; GERHARD, M.; KAISER, W.; GLAAB, J.; CONRATH, U.; SEEHAUS, K.; HERMS, S. Physiological effects of the strobilurin fungicide F 500 on plants. In: DEHNE, H.W.; GISI, U.; KUCK, K.H.; RUSSELL, P.E.; LYR, H. (Ed.). Modern fungicides and antifungal compounds III. Andover, 2003. p. 61-74.

KOZLOWSKI, L. A.; SIMOES, D. F. M.; SOUZA, C. D. de; TRENTO, M. Efeito fisiológico de estrobilurina $F 500^{\circledR}$ no crescimento e rendimento do feijoeiro. Revista Acadêmica: Ciências Agrárias e Ambientais, v. 7, n. 1, p. 41-54, 2009.

LIMA, J. D.; MORAES, W. S. da; SILVA, S. H. M-G. da. Respostas fisiológicas em mudas de bananeira tratadas com estrobilurinas. Semina: Ciências Agrárias, Londrina, v. 33, n. 1, p .77-86, 2012. DOI: http://dx.doi.org/10.5433/1679-0359.2012v33n1p77

MARTINS, L. D.; TOMAZ, M. A.; SOUZA, A. F. de; JESUS JÚNIOR, W. C. de; RODRIGUES, W. N. Influência da aplicação de ciproconazol + tiametoxam no crescimento de mudas de Coffea canephora Pierre ex A. Froehner. Revista de Ciências Agrárias, v. 34, n. 1, p. 220-228, 2011.

OLIOSI, G.; GILES, J. A. D.; RODRIGUES, W. P.; RAMALHO, J. C.; PARTELLI, F. L. Microclimate and development of Coffea canephora cv. Conilon under 
different shading levels promoted by Australian cedar (Toona ciliata M. Roem. var. Australis). Australian Journal of Crop Science, v. 10, n. 4, p. 528-538, 2016. DOI: https://doi.org/10.21475/ajcs.2016.10.04.p7295x

PAIVA, R. F. de; MENDES, A. N. G.; CARVALHO, G. R.; REZENDE, J. C. de; FERREIRA, A. D.; CARVALHO, A. M. de. Comportamento de cultivares de cafeeiros $C$. arabica L. enxertados sobre cultirar "Apoatã" IAC 2258 (Coffea canephora). Ciência Rural, Santa Maria, v. 42, n. 7 , p. 1155-1160, 2012. DOI: http://dx.doi.org/10.1590/S0103-84782012000700003.

PAULO JÚNIOR, J.; FAGAN, E. B.; CORREA, L. T.; SOARES, J. N.; PEREIRA, I. S.; SILVA, L. G. Resposta fisiológica de mudas de café à aplicação foliar de estrobilurina-piraclostrobina e silício. Cerrado Agrociências, v. 4, n. 1, p. 42-57, 2013.

PREZOTTI, L. C.; BRAGANÇA, S. M.; MARTINS, A. G.; LANI, J. A. Nutrição, calagem e adubação. In: FONSECA, A.; SAKIYMA, N.; BORÉM, A. (Eds.). Café conilon: do plantio a colheita. Viçosa: UFV, 2015. Cap.5, p.89-113.

PREZOTTI, L. C.; GOMES, J. A.; DADALTO, G. G.; OLIVEIRA, J. A. Manual de recomendação de calagem e adubação para o Estado do Espírito Santo - $5^{\text {a }}$ aproximação. Vitória: SEEA/INCAPER/ CEDAGRO, 2007. 305p.

RAMOS, A. R. P.; AMARO, A. C. E.; MACEDO, A. C.; SUGAWARA, S. A. de; EVANGELISTA, R. M.; RODRIGUES, J. D.; ONO, E. O. Qualidade de frutos de tomate 'giuliana' tratados com produtos de efeitos fisiológicos. Semina: Ciências Agrárias, Londrina, v. 34, http://dx.doi.org/10.5433/1679-
0359.2013v34n6Supl1p3543
SISTEMA DE AGROTÓXICOS FITOSSANITÁRIOS (AGROFIT) - MINISTÉRIO DA AGRICULTURA, PECUÁRIA E ABASTECIMENTO (MAPA). Agricultura. 2017.2 Disponível em: http://agrofit.agricultura.gov.br/agrofit_cons/principal_ag rofit_cons. Acesso em: 06 mar. 2017.

SILVA, F. A. S.; AZEVEDO, C. A. V. Comparison of means of agricultural experimentation data through diferente tests using the software Assistat. Academic Journals, v. 11, n. 37, p. 3527-3531, 2016. DOI: 10.5897/AJAR2016.11523

SOARES, A. R.; EVERALDO, C. M.; RENA, A. B.; SOARES, A. A. Irrigação e fisiologia da floração em cafeeiros adultos na região da zona da mata de Minas Gerais. Acta Scientiarum Agronomy, Maringá, v. 27, n. 1, p. 117-125, 2005.2 DOI: http://dx.doi.org/10.4025/actasciagron.v27i1.2128

SOARES, L. H.; FAGAN, E. B.; CASAROLI, D.; ANDRADE, D. M. de; SOARES, A. L.; MARTINS, K. V.; ROCHA, F. J. da. Aplicação de diferentes estrobilurinas na cultura da soja. Revista da FZVA, v. 18, n. 1, p. 78-97, 2011.

VENÂNCIO, W. S.; RODRIGUES, M. A. T.; BEGLIOMINI, E.; SOUZA, N. L. de. Physiological effects of strobilurin fungicides on plants. Publication UEPG Ciências Exatas e da Terra, v. 9, n. 3, p. 59-68, 2003. DOI: ttp://dx.doi.org/10.5212/publicatio.v9i03.814

ZAMBOLIM, L.; SOBREIRA, D. G.; SOUZA, A. F. de; COSTA, H. Manejo integrado das doenças do conilon (Coffea canephora). In: ZAMBOLIM, L. (Ed.). Tecnologias para produção do café Conilon. Viçosa: UFV, 2009. Cap.1, p.1-45. 